\title{
Salud mental, abuso de sustancias y trastornos duales en el embarazo: Tasas de prevalencia y tratamiento en un país desarrollado
}

\section{Substance use, mental health and dual disorders on pregnancy: Results of prevalence and treatment rates in a developed country}

\begin{abstract}
Rodrigo Carmona Camacho*, Nayara lópez Carpintero**, María Luisa Barrigón*,********, Cristina Ruiz Nogales*, Inés Menéndez*, Montserrat Sánchez Alonso*, Irene Caro Cañizares*, Juan José Hernández Aguado**, Benjamin Le Cook***, Margarita Alegría****, Ricardo Saviron Cornudella $* * * * *$, Javier PlazA $* * * * * *$, Enrique BaCA-García*,********************.
\end{abstract}

\footnotetext{
* Departamento de Psiquiatría. Fundación Jiménez Díaz, Madrid, España.

** Departamento de Obstetricia y Ginecología. Hospital Infanta Leonor, Madrid, España.

*** Departamento de Psiquiatría, Escuela de Medicina de Harvard; Health Equity Research Lab.

Departamento de Psiquiatría, Cambridge Health Alliance. Cambridge, EE. UU.

**** Departamento de Psiquiatría, Escuela de Medicina de Harvard; Disparities Research Unit, Departamento de Medicina,

Hospital General de Massachusetts y Escuela de Medicina de Harvard, Boston, EE. UU.

***** Departamento de Obstetricia y Ginecología. Hospital General de Villalba, Madrid, España.

******* Departamento de Obstetricia y Ginecología. Fundación Jiménez Díaz, Madrid, España.

******* Departamento de Psiquiatría. Hospital Universitario Rey Juan Carlos, Móstoles. España; Departamento de Psiquiatría. Hospital General de Villalba, Madrid. España; Departamento de Psiquiatría. Hospital Universitario Infanta Elena, Valdemoro, España.

******** Departamento de Psiquiatría. Universidad Autónoma de Madrid, Madrid, España.

********* CIBERSAM (Centro de Investigación Biomédica en Red Salud Mental), Carlos III Instituto de Salud, Madrid, España.

********** Universidad Católica del Maule, Talca, Chile.

************ Departamento de Psiquiatría. Centre Hospitalier Universitaire de Nîmes, Francia.
}

\section{Resumen}

El tabaquismo y el consumo de sustancias durante el embarazo son importantes causas prevenibles de morbimortalidad, teniendo una relación bidireccional y deletérea con la salud mental de la madre y el niño. Como parte de la iniciativa WOMAP (Woman Mental Health and Addictions on Pregnancy), se estudiaron 2.014 embarazadas buscando describir la prevalencia de trastornos mentales y por uso de sustancias concurrentes, las tasas de tratamiento y los diagnósticos y la gravedad. Las participantes fueron evaluadas con la escala AC-OK y se les preguntó sobre sus hábitos tabáquicos y uso de servicios de salud mental/sustancias. De las participantes, 170 mujeres resultaron positivas para un trastorno mental y por uso de sustancias concurrentes ( $\geq 2$ positivos a la subescala AC-OK-Salud Mental, $\geq 1$ positivos a la subescala AC-OK-Sustancias y/o fumar más de una vez al mes y no estar en tratamiento) y fueron evaluadas en profundidad mediante una batería de escalas (Patient Health

\begin{abstract}
Smoking and substance use during pregnancy are major preventable causes of mortality and morbidity, having a bidirectional and deleterious relationship with the mental health of the mother and child. As part of the WOMAP (Woman Mental Health and Addictions on Pregnancy) initiative, our study aimed to describe the prevalence of co-occurring mental illness and substance use problems, diagnoses and severity of those considered at risk and rates of treatment. A screening of 2,014 pregnant women was done using the AC-OK scale and they were asked about their smoking habits and services use for mental health/substance abuse. Of these, 170 women were considered at risk of co-occurring mental illness and substance use problems ( $\geq 2$ positive responses to the AC-OK-Mental Health subscale, $\geq 1$ positive response to the AC-OK-Substance Abuse subscale and/or smoking more than once a month and no use of specialized
\end{abstract}


Questionnaire [PHQ-9], General Anxiety Disorder [GAD-7], Post-traumatic stress disorder Checklist [PCL-5], Alcohol Use Disorders Identification Test [AUDIT], Drug Abuse Screening Test [DAST] y Fagerström). En el último año, 614 mujeres $(30,5 \%)$ fumaron tabaco $(42,5 \%$ diariamente) y el $9,8 \%$ fueron positivas para problemas por uso de sustancias y salud mental según la AC-OK. Solo el 11,1\% había recibido tratamiento en los tres meses previos y solo un $13,6 \%$ tenía una cita en el siguiente mes. De las 170 pacientes evaluadas secundariamente, 62(36,5\%) presentaron al menos depresión moderada, 35(20,6\%) al menos ansiedad moderada, 32(18,8\%) fueron positivas a la PCL-5, y 37 de las 88 que reconocieron uso de alcohol puntuaron por encima del umbral en AU$\operatorname{DIT}(\geq 3)$.

En conclusión, la combinación de una prevalencia significativa junto con bajas tasas de tratamiento, remarcan la necesidad de mecanismos de detección efectivos en la atención habitual, permitiendo una intervención temprana.

Palabras clave: Atención perinatal; salud mental perinatal; patología dual; tabaquismo; consumo de drogas; cribado. services) and were assessed with a more extensive battery of measures (Patient Health Questionnaire [PHQ-9], General Anxiety Disorder [GAD-7], Posttraumatic stress disorder [PTSD] Checklist for DSM-5 [PCL-5], Alcohol Use Disorders Identification Test [AUDIT], Drug Abuse Screening Test [DAST] and Fagerström).

In the last year, 614 women $(30.5 \%)$ smoked tobacco ( $42.5 \%$ daily) and $9.8 \%$ were positive for both substance use and mental illness per the AC-OK. Only $11.1 \%$ of them received specific treatment in the previous three months while another $13.6 \%$ were scheduled to attend services in the following month. From the subsample assessed in depth, 62(36.5\%) endorsed at least moderate depression, 35(20.6\%) endorsed at least moderate anxiety, 32(18.8\%) endorsed PTSD on the PCL, and 37 out of 88 alcohol users scored above the threshold in AUDIT $(\geq 3)$.

In conclusion, high prevalence and low treatment rates suggest that effective detection mechanisms should be integrated into usual care, allowing for early interventions.

Keywords: Perinatal care; perinatal mental health; dual disorders; smoking; drug use; screening.

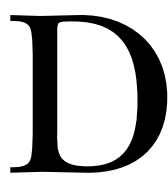
urante las primeras semanas de gestación, un adecuado seguimiento de los factores de riesgo relacionados con las complicaciones fetales, mediante una adecuada anamnesis, es fundamental para un seguimiento óptimo. Casi todos los obstetras examinan antecedentes como preeclampsia previa o parto prematuro en un nuevo embarazo para prevenir y proporcionar un diagnóstico temprano de recurrencias. Sin embargo, a pesar de que el consumo de sustancias, especialmente el tabaquismo, es aún más frecuente (Lange, Probst, Rehm y Popova, 2018), y tiene un impacto crucial tanto a corto como a largo plazo, suele estar infradiagnosticado y no siempre forman parte de la evaluación sistemática de todos los obstetras (Hankin, McCaul y Heussner, 2000). Además, la no detección del consumo de sustancias puede provocar complicaciones gestacionales, como parto prematuro, rotura prematura de membranas o restricción del crecimiento fetal (Cnattingius, 2004; Dahlin, Gunnerbeck, Wikstrom, Cnattingius y Edstedt Bonamy, 2016; England, Benjamin y Abenhaim, 2013; Gouin, Murphy y Shah, 2011; Ko et al., 2014) y, por tanto, es una causa potencialmente prevenible de complicaciones. El consumo de sustancias se vuelve aún más problemático si se tiene en cuenta que las mujeres que consumen sustancias durante el embarazo suelen consumir más de una sustancia ilícita, multiplicando así el riesgo de enfermedad fetal (Forray y Foster, 2015). La exposición a drogas ilícitas durante el embarazo también se ha relacionado con efectos estructurales en el feto y una variedad de consecuencias neuroconductuales durante la niñez y posteriormente (Holbrook y Rayburn, 2014).

$\mathrm{Al}$ enfrentarnos al consumo de sustancias en mujeres embarazadas, debemos considerar las interrelaciones en- tre malformaciones congénitas específicas, prematuridad, restricción del peso al nacer, mortinatos y síndrome de abstinencia fetal posterior o muerte neonatal, y otras complicaciones menos frecuentes (Dahlin et al., 2016; Gauthier, Guidot, Kelleman, McCracken y Brown, 2016; Ko et al., 2014; Pereira, Da Mata, Figueiredo, de Andrade y Pereira, 2017; Pineles, Hsu, Park y Samet, 2016). Las dos complicaciones más relevantes del tabaquismo son la prematuridad y el bajo peso al nacer. En ambos casos, el cese del tabaquismo durante el primer trimestre iguala el riesgo al de no fumadoras (Blatt, Moore, Chen, Van Hook y DeFranco, 2015). Estas complicaciones dependen de la dosis (Cnattingius, 2004). Lo mismo ocurre con las sustancias lícitas, con estudios que describen complicaciones físicas (Cook et al., 2017) y problemas conductuales y psiquiátricos relacionados con el consumo de tabaco (Ekblad, Gissler, Lehtonen y Korkeila, 2010; Tiesler y Heinrich, 2014) y alcohol (Donald et al., 2015; Sarman, 2018).

Esto es de particular interés dado que algunas mujeres no modifican su patrón de consumo de sustancias antes de la confirmación del embarazo (Holbrook y Rayburn, 2014). Por tanto, la prevalencia de tabaquismo durante el embarazo es similar a la de la población general (Cnattingius, 2004). Un metaanálisis reciente estima la prevalencia del tabaquismo durante el embarazo en España de 26\% (Lange et al., 2018), una probable subestimación del problema, dada la notoria falta de información (Garg et al., 2016). Un importante problema de salud pública en las mujeres embarazadas son los problemas perinatales de salud mental, relacionados de forma bidireccional y perjudicial con el consumo de sustancias. Los estudios de prevalencia de depresión y ansiedad en varios países han descrito un rango de entre $10-30 \%$ en mujeres embarazadas 
(Austin, Priest y Sullivan, 2008; Bayrampour, Hapsari y Pavlovic, 2018; Fairbrother, Janssen, Antony, Tucker y Young, 2016; Martinez-Paredes y Jacome-Perez, 2019; Woody, Ferrari, Siskind, Whiteford y Harris, 2017). De manera similar al uso de sustancias, los problemas perinatales de salud mental se asocian con varios resultados adversos del embarazo (Kramer et al., 2009) y trastornos del neurodesarrollo y problemas de salud mental en niños (Kingston, Tough y Whitfield, 2012).

A pesar de varias iniciativas que revelan una identificación insuficiente (Hankin et al., 2000) y que recomiendan un cribado sistemático, no existen pautas claras universales y, en consecuencia, las afecciones de salud mental durante el embarazo todavía están infradiagnosticadas (Bayrampour et al., 2018). Sin embargo, pocos estudios han analizado la coexistencia de problemas de salud mental y uso de sustancias en esta población. La falta de servicios para tratar la salud mental perinatal en muchos entornos sanitarios evidencia la necesidad de mejorar la detección y la atención de estas mujeres (Howard, Piot y Stein, 2014).

Se han identificado diferentes barreras al abordaje adecuado de la salud mental durante el embarazo (Bayrampour et al., 2018). Una forma de superar las barreras para la identificación y la atención es el desarrollo de cuestionarios de cribado de fácil uso para obstetras y matronas. La herramienta de cribado AC-OK es un instrumento útil validado en español y con buenas propiedades psicométricas para el cribado rutinario de problemas de salud mental y de consumo de sustancias en entornos clínicos (Chavez et al., 2017).

En este estudio, utilizamos datos obtenidos del proyecto de investigación WOMAP (Woman Mental Health and Addictions on Pregnancy), un estudio diseñado para probar la viabilidad, aceptabilidad y eficacia de dos intervenciones diferentes para mujeres embarazadas con problemas de tabaquismo, benzodiazepinas u otros problemas de uso de sustancias y/o trastornos de salud mental. Nuestros objetivos eran: 1) describir la prevalencia de consumo de sustancias y problemas de salud mental utilizando el cuestionario AC-OK, incluyendo la prevalencia del tabaquismo; 2) describir si estas mujeres recibieron o no tratamiento, y 3) identificar los trastornos de salud mental y diagnóstico de uso de drogas y la gravedad en una submuestra de mujeres consideradas elegibles para el ensayo clínico WOMAP.

\section{Métodos}

\section{Entorno y participantes}

Entre julio de 2016 y diciembre de 2019, se cribaron a 2014 mujeres embarazadas. Las participantes fueron seleccionadas entre las mujeres cuyo embarazo era de menos de 26 semanas, mayores de 18 años y que acudían a revisiones obstétricas en cinco hospitales de la zona metropolitana de Madrid (España): Fundación Jiménez Díaz (zona urbana de Madrid), el Hospital Infanta Leonor (zona urbana de Madrid), Hospital Universitario del Tajo (Aranjuez), Hospital General de Villalba (Villalba) y Hospital Infanta Elena (Valdemoro). Los cinco hospitales participantes abarcan un área de salud de más de 1300000 habitantes de diversos perfiles socioeconómicos.

Las participantes fueron abordadas de dos formas: in situ, cuando la mujer finalizaba la visita obstétrica, o por teléfono, después de que el obstetra a cargo obtuviese la autorización de la paciente para contactarla. En ambos casos, el estudio fue explicado a las participantes por los asistentes de investigación, y la entrevista de selección se administró a quienes aceptaron participar. La atención habitual a las embarazadas en los hospitales de reclutamiento comienza con una primera cita entre las 8-12 semanas de gestación en la que se realiza una anamnesis detallada y una ecografía fetal. También se evalúan los resultados analíticos del primer trimestre (semana 10-12) y se examinan las anomalías cromosómicas. En las semanas 20, 28 y 32-34 de gestación, se realizan ecografías morfológicas que miden el crecimiento fetal y se evalúan los resultados analíticos de cada trimestre. Posteriormente, en la semana 38-39, se realiza una ecografía de estática fetal y valoración del crecimiento fetal y líquido amniótico. Desde las semanas 40-41, se realizan controles fetales y ecográficos para evaluar el perfil biofísico.

\section{Valoración}

Las participantes fueron evaluadas primero para identificar a aquellas consideradas en riesgo de problemas de salud mental/uso de sustancias coexistentes. A las que dieron positivo en el cribado se les ofreció participar en el ensayo clínico WOMAP y luego se les evaluó con un cuestionario más completo. Los datos se recopilaron y almacenaron mediante la interfaz para médicos MeMind (www. memind.net), una aplicación en línea desarrollada para fusionar diferentes fuentes de datos, incluidos los datos de médicos y pacientes (Barrigon et al., 2017).

\section{Valoración del cribado}

Hubo 2014 mujeres que aceptaron ser cribadas y fueron evaluadas por problemas de salud mental (SM), abuso de sustancias (AS) y coexistencia de problemas de salud mental/uso de sustancias (patología dual) con la prueba ACOK (Chavez et al., 2017; Cherry y Dillon, 2013). El AC-OK es un cuestionario fácil de usar, validado en poblaciones de habla hispana en España y EE.UU., diseñado para ser un detector útil de problemas de salud mental y de abuso de sustancias. El AC-OK incluye 15 ítems, nueve elementos relacionados con la salud mental y seis elementos relacionados con el abuso de sustancias. La versión en español tiene buenas propiedades psicométricas, con buena consistencia interna (cribado de salud mental $[\alpha=, 82]$; cribado 
de abuso de sustancias $[\alpha=, 90])$ y excelente sensibilidad y especificidad. Se seleccionó un punto de corte de dos o más respuestas positivas para la subescala de salud mental y una o más para la subescala de consumo de sustancias (Chavez et al., 2017). Aquellas mujeres que respondieron «sí» a las preguntas 9 o 10 del AC-OK respecto al deseo de morir y la conducta suicida, fueron evaluadas para el riesgo de suicidio en el mes anterior con la Escala Paykel de Suicidio (Paykel, Myers, Lindenthal y Tanner, 1974) y derivadas a una evaluación psiquiátrica inmediata como parte de un protocolo de seguridad en caso de responder afirmativamente a las preguntas 4 o 5 de Paykel (i.e., tener un plan o intento de suicidio). Este cuestionario consta de cinco preguntas sobre el suicidio que incluyen el hartazgo de vivir, los deseos de morir, la ideación suicida, los planes suicidas y los intentos de suicidio.

Además, se preguntó a las mujeres sobre el consumo de cigarrillos en los últimos 12 meses (sí/no) y, entre las que respondieron afirmativamente, con qué frecuencia fumaban (número de días semanales que fumaban cigarrillos). También se les preguntó sobre los servicios de salud mental y/o abuso de drogas/alcohol utilizados en los últimos tres meses y si tenían una cita programada para el próximo mes. Finalmente, se registraron características sociodemográficas: edad, país de origen, grupo racial y nivel de estudios.

\section{Evaluación para mujeres en riesgo}

Las mujeres se consideraron en riesgo (y por tanto fueron incluidas en el ensayo clínico) si: 1) tenían dos o más respuestas positivas a la subescala AC-OK-subescala de salud mental (AC-OK-MH), 2) tenían una o más respuestas positivas a la AC-OK-subescala de abuso de sustancias (ACOK-SA) y/o informó que fumaba más de una vez al mes, 3) no eran usuarias de servicios especializados, definido como no tener una cita en el mes siguiente y no haber visto a un médico en los últimos tres meses y 4) respondieron $\mathrm{NO}$ a las preguntas 4 y 5 (Paykel et al., 1974) en caso de haberse administrado la Escala Paykel de Suicidio. Los criterios de exclusión para acceder al ensayo clínico fueron: 1) haber recibido un diagnóstico de trastorno psicótico o relacionado con el trastorno bipolar o 2) tener incapacidad para dar consentimiento, determinado por no poder responder a las preguntas del propósito o proceso del estudio.

Debido a los protocolos de ensayos clínicos del estudio WOMAP, solo las mujeres elegibles $(n=170)$ fueron evaluadas con una batería más extensa de cuestionarios de salud mental y uso de sustancias. Para la salud mental, utilizamos el Cuestionario de la salud del paciente (PHQ-9) que aborda los nueve criterios de diagnóstico del DSM-IV para el trastorno depresivo mayor (Kroenke, Spitzer y Williams, 2001), el Cribado de 7 ítems del trastorno de ansiedad general (GAD-7) para la ansiedad (Spitzer, Kroenke, Williams y Lowe, 2006), y la Lista de verificación del trastorno por estrés postraumático (PCL-5), una medida de autoin- forme para los 17 síntomas de TEPT del DSM-IV (Blanchard, Jones-Alexander, Buckley y Forneris, 1996). Para el uso de sustancias, se administraron los instrumentos Cuestionario de Identificación de los Trastornos debidos al Consumo de Alcohol (AUDIT), un cribado desarrollado por la Organización Mundial de la Salud (OMS) (Bohn, Babor y Kranzler, 1995); la Prueba de Detección de Abuso de Drogas (DAST), un instrumento breve de autoinforme diseñado para la detección de trastornos por abuso y dependencia de drogas (Yudko, Lozhkina y Fouts, 2007); y el Test de Fagerström de dependencia de la nicotina, un instrumento de seis ítems que evalúa la cantidad de consumo de cigarrillos, la compulsión de fumar y la dependencia del tabaquismo (Heatherton, Kozlowski, Frecker y Fagerstrom, 1991). Además, se les preguntó a las mujeres con cribado positivo respecto del número de días en el último mes que consumieron las siguientes sustancias: alcohol, opioides, barbitúricos, benzodiazepinas, cocaína, anfetaminas, cannabis, alucinógenos e inhalantes.

\section{Análisis estadístico}

Se realizó un estudio descriptivo de la muestra total con respecto a las variables demográficas con medias y desviaciones estándar o porcentajes, según proceda. Las tasas de cribado positivo al AC-OK y sus subescalas se calcularon para la muestra total y se compararon por nivel de estudios y grupo racial utilizando pruebas de chi-cuadrado. El tabaquismo se describió en la muestra total con porcentajes. Para la submuestra evaluada en profundidad, se calcularon puntuaciones de los cuestionarios.

\section{Consideraciones éticas y protección de datos}

El estudio se realizó conforme a la Declaración de Helsinki y fue aprobado por el Comité Ético de Investigación Clínica de la Fundación Jiménez Díaz (Ref. 2015/43). Después de una descripción completa del estudio, todas las participantes cribadas dieron su consentimiento informado.

En cuanto a la protección de datos, se restringió a los investigadores el acceso al interfaz de usuario clínico de MeMind. Solo el investigador principal, los médicos y los investigadores, mediante nombre de usuario y contraseña, tenían acceso a la información identificable. Los datos proporcionados por el médico fueron encriptados por Secure Socket Layer/Transport Layer Security (SSL/TLS) entre el ordenador del investigador y el servidor. Los datos se almacenaron en un servidor externo creado con fines de investigación. Los datos se cifraron mediante el algoritmo AES-256 estándar de la industria. Asimismo, un auditor externo garantizó que las medidas de seguridad cumplían con la Ley Orgánica de Protección de Datos (Gobierno de España, Ley Orgánica 3/2018 de Protección de Datos Personales y garantía de los derechos digitales, 2018) en un nivel de protección alto. 


\section{Resultados}

\section{Descripción de la muestra total}

La muestra total fue de 2014 mujeres con edades de 33,0 \pm 5,6 años (rango 18-46). El 67,6\% de las mujeres nacieron en España, el 19,8\% en América del Sur o Central, el 6,2\% en el resto de Europa, el 2,5\% en Marruecos y el 3,9\% en otros países no europeos.

En cuanto a la identificación de grupo racial, el 75,4\% (1518) de las mujeres que proporcionaron información (1965) se identificaron como blancas; y el 14,3\% (287) declaró su origen étnico como latinoamericano. Otros grupos raciales minoritarios incluyeron: $1,9 \%(\mathrm{n}=39)$ gitano, 2,3\% $(\mathrm{n}=46)$ árabe, $2,2 \%(\mathrm{n}=45) \mathrm{raza} /$ etnia mestiza, $1 \%$ $(\mathrm{n}=20)$ afroamericano y $0,5 \%(\mathrm{n}=10)$ asiático. En cuanto a estudios, 880 mujeres $(43,7 \%)$ tenían título universitario o superior, la mitad de las mujeres $(49,6 \%$; $\mathrm{n}=998)$ completaron estudios secundarios o la escuela técnica profesional y 129 mujeres $(6,4 \%)$ completaron estudios primarios.

En cuanto a la salud mental y el abuso de sustancias, de las 2014 mujeres cribadas, el 9,8\% (198) tuvo dos o más respuestas positivas a la subescala AC-OK-MH y una o más respuestas positivas a la subescala AC-OK-SA y, por tanto, fueron consideradas en alto riesgo de tener problemas coexistentes de salud mental/consumo de sustancias, una tasa que aumenta a 17,1\% (344) al añadir tabaquismo en el último año a la AC-OK como criterio para la consideración de riesgo. Para cada subescala, los porcentajes de ítems positivos se muestran en la Figura 1. Se observaron diferencias por nivel de estudios, con un $14,7 \%(\mathrm{n}=19)$ de las mujeres consideradas en alto riesgo de un trastorno coexistente de aquellas con estudios primarios, en comparación con $11,2 \%(n=112)$ de aquellas con estudios secundarios y $7,5 \%$ de aquellas con estudios universitarios $(n=66)(p=$ ,004). Estas diferencias permanecen al agregar tabaquismo al AC-OK como criterio para la consideración de estar en riesgo: $24 \%(\mathrm{n}=31)$ con estudios primarios, $19,9 \%(\mathrm{n}=$
119) con estudios secundarios y $12,7 \%(n=112)$ con estudios universitarios $(\mathrm{p}<, 001)$.

Con respecto a la salud mental, el $32 \%$ de las mujeres ( $\mathrm{n}=645)$ respondió afirmativamente a dos o más ítems, mientras que el $15 \%(\mathrm{n}=302)$ respondió afirmativamente a uno o más ítems sobre el abuso de sustancias. No se encontraron diferencias en el AC-OK-SA por raza o nivel de estudios. Un mayor porcentaje de mujeres con solo estudios primarios dieron positivo en el AC-OK-MH (43,4\%; $\mathrm{n}=56)$ en comparación con el 36,7\% ( $\mathrm{n}=366)$ entre las que tenían estudios secundarios o escuela técnica profesional y el $25 \%(n=220)$ con estudios universitarios $(\mathrm{p}<, 001)$. Al evaluar las diferencias por raza, las que se autoidentificaron como asiáticas $(20 \%)$, gitanas $(20,5 \%)$, blancas $(30,4 \%)$ o latinoamericanas $(34,8 \%)$ tuvieron tasas positivas más bajas en el AC-OK-MH en comparación con las afroamericanas $(55,0 \%)$, mestizas $(46,7 \%)$ o árabes $(41,3 \%)(\mathrm{p}=, 006)$.

De las 198 considerados en riesgo según la AC-OK, 22 $(11,1 \%)$ recibieron tratamiento en un centro de salud mental o drogadicción en los tres meses anteriores y 27 $(13,6 \%)$ tenían cita para acudir a los servicios en el mes siguiente.

A las 99 mujeres que respondieron «sí» a las preguntas 9 o 10 de la AC-OK, sobre el deseo de morir y conducta suicida, se les preguntó sobre el riesgo de suicidio mediante la Escala Paykel de Suicidio. De ellas, dos (2\%) informaron haber tenido un intento de suicidio, seis $(6,1 \%)$ habían ideado un plan de suicidio, 21 (21,2\%) tenían pensamientos suicidas, $30(30,3 \%)$ expresaron deseos de morir y 28 $(29,3 \%)$ reportaron hartazgo de vivir. Del total de la muestra, 614 mujeres $(30,5 \%)$ habían fumado tabaco durante el último año (Tabla 1).

\section{Descripción de mujeres en riesgo}

Ciento setenta mujeres $(8,4 \%)$ que cumplieron los criterios de inclusión del estudio fueron evaluadas con una

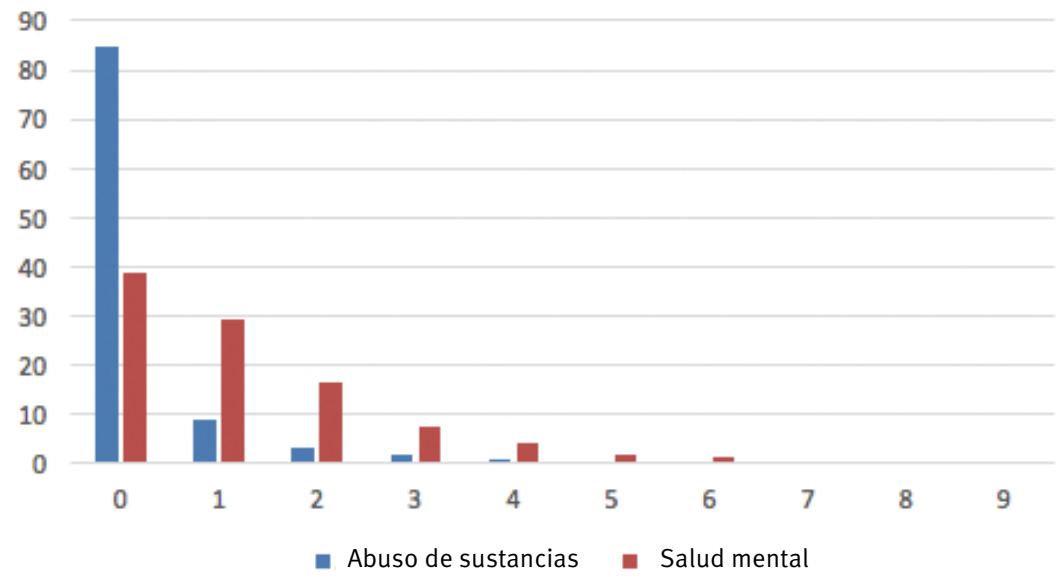

Figura 1. Porcentaje de mujeres por el número de respuestas afirmativas en AC-OK en las subescalas SA (6 ítems) y MH ( 9 ítems). 
Tabla 1. Frecuencia de tabaquismo $(n=614)^{1}$.

\begin{tabular}{lcc}
\hline Frecuencia & N & Porcentaje \\
\hline A diario & 261 & $42,5 \%$ \\
5 a 6 días/semana & 10 & $1,6 \%$ \\
3 a 4 días/semana & 13 & $2,1 \%$ \\
1 a 2 días/semana & 18 & $2,9 \%$ \\
2 a 3 días/mes & 10 & $1,6 \%$ \\
Menos de una vez al mes & 296 & $48,2 \%$ \\
\hline
\end{tabular}

Nota. ${ }^{1}$ Faltan los datos de seis pacientes (1\%).

Tabla 2. Resultados de los cuestionarios de salud mental y abuso de sustancias $(n=170)$.

\begin{tabular}{|c|c|c|}
\hline & $\mathbf{N}$ & Porcentaje \\
\hline \multicolumn{3}{|l|}{ Depresión (PHQ-9)a } \\
\hline Negativo $(0-4)$ & 40 & $23,5 \%$ \\
\hline Depresión leve (5-9) & 68 & $40,0 \%$ \\
\hline Depresión moderada (10-14) & 41 & $24,1 \%$ \\
\hline Depresión moderadamente severa (15-19) & 16 & $9,4 \%$ \\
\hline Depresión severa (20-27) & 5 & $2,9 \%$ \\
\hline Positivo (PHQ-9 $\geq 10$ ) & 62 & $36,5 \%$ \\
\hline \multicolumn{3}{|l|}{ Ansiedad generalizada (GAD-7) } \\
\hline Negativo $(0-4)$ & 70 & $41,2 \%$ \\
\hline Ansiedad leve (5-9) & 65 & $38,2 \%$ \\
\hline Ansiedad moderada (10-14) & 22 & $12,9 \%$ \\
\hline Ansiedad severa $(15-21)$ & 13 & $7,6 \%$ \\
\hline Positivo (GAD-7 $\geq 10$ ) & 35 & $20,6 \%$ \\
\hline \multicolumn{3}{|l|}{ TEPT $(\mathrm{PCL})^{\mathrm{b}}$} \\
\hline Negativo & 138 & $81,2 \%$ \\
\hline Positivo (PCL $\geq 33$ ) & 32 & $18,8 \%$ \\
\hline \multicolumn{3}{|l|}{ Uso de alcohol (AUDIT)c } \\
\hline Negativo & 51 & $58,0 \%$ \\
\hline Positivo (AUDIT $\geq 3$ ) & 37 & $42,0 \%$ \\
\hline \multicolumn{3}{|l|}{ Abuso de sustancias (DAST) ${ }^{d}$} \\
\hline Negativo & 37 & $21,8 \%$ \\
\hline Positivo (DAST $\geq 3$ ) & 0 & 0 \\
\hline
\end{tabular}

Nota. a: faltan los datos para una mujer; b: 5 mujeres no informaron eventos traumáticos; c: 82 mujeres no tuvieron consumo de alcohol; d: 133 mujeres no tuvieron consumo de sustancias.

Tabla 3. Resultados del cuestionario de Fagerström (solo se evaluaron aquella que informaron uso en el último año [87]).

\begin{tabular}{lcc}
\hline & N & Porcentaje \\
\hline Dependencia muy baja & 51 & $58,6 \%$ \\
Dependencia baja & 21 & $24,1 \%$ \\
Dependencia moderada & 10 & $11,5 \%$ \\
Dependencia alta & 4 & $4,6 \%$ \\
Dependencia muy alta & 1 & $1,1 \%$ \\
\hline
\end{tabular}

batería de cuestionarios en profundidad. Las Tablas 2 y 3 muestran los resultados.

Cuando se les preguntó sobre el uso de sustancias durante el mes anterior, 23 mujeres informaron consumo de alcohol (de 12 mujeres que bebieron un solo día hasta un máximo de una mujer que bebió a diario), ocho consumieron sedantes (cinco de ellas durante todo el mes, otra durante 23 días, y dos mujeres más de 4 y 3 días cada una), cinco mujeres fumaron cannabis (tres a diario y dos veces durante el mes); y una mujer informó haber consumido cocaína durante 7 días. El resto de sustancias no fueron utilizadas por las 170 mujeres evaluadas.

\section{Discusión}

El análisis de la muestra de 2014 mujeres embarazadas arrojó cifras de consumo de tabaco superiores a las de un metaanálisis reciente que estimaba una prevalencia de tabaquismo durante el embarazo del 26\% (Lange et al., 2018). Casi un tercio de nuestra muestra $(30,5 \%$ de las mujeres) fumó tabaco en el último año, y 261 de las fumadoras (42,5\% de las fumadoras) declararon haber fumado a diario. En España, un estudio reciente encontró una prevalencia de consumo de sustancias en cada uno de los trimestres del embarazo del 21,2\%, $18,5 \%$ y $13,3 \%$ respectivamente para el tabaco, $40,7 \%, 23,1 \%$ y $17,1 \%$ para el alcohol y $4,8 \%, 1,9 \%$ y $1,2 \%$ para cannabis (Blasco-Alonso et al., 2015). Al igual que este aparente cese del tabaquismo progresivo cuando se confirma el embarazo, nuestra muestra reveló que, de las 614 mujeres que afirmaron en el momento de la evaluación haber fumado en el último año, el $48,2 \%$ informaron haber fumado menos de una vez al mes.

Una contribución relevante de este estudio a la escasa información existente en la literatura sobre problemas concurrentes en mujeres embarazadas es que el 9,8\% (198 mujeres) fueron consideradas en riesgo de una patología dual de enfermedad mental y trastorno por uso de sustancias. Ademas, si se considera a las que reportaron consumo de tabaco en el último año, el 17,1\% estaban en riesgo.

$\mathrm{Si}$ bien en la literatura se ha informado sobre el abuso de sustancias en mujeres embarazadas (Blasco-Alonso et al., 2015; Chang et al., 2011), rara vez se ha descrito la patología dual. De la submuestra de 170 mujeres elegibles para el ensayo WOMAP que fueron evaluadas en profundidad, el 36,5\% fueron positivas a depresión al menos moderada, el $20,6 \%$ fueron positivas a ansiedad al menos moderada, el $18,8 \%$ fueron positivas a la escala de TEPT y 37 puntuaron por encima del umbral en AUDIT (21,8\%). Estas tasas, nuevamente, son clínicamente relevantes y coinciden con las obtenidas en estudios previos que describen tasas de hasta el $20 \%$ en depresión y ansiedad durante el embarazo (Austin et al., 2008; Bayrampour et al., 2018). Las tasas significativas de prevalencia de salud mental, abuso de sustancias y patologías duales encontrados en la muestra de 
mujeres embarazadas con resultados positivos en el AC-OK evidencian la necesidad de explorar esfuerzos adicionales para tratar las patologías duales durante los servicios de atención inicial al embarazo, más cuando el 4,91\% (99) de las mujeres cribadas admitieron tener pensamientos de morir o ideación suicida.

Cabe señalar que solo una pequeña proporción de las pacientes identificadas con riesgo de trastornos duales mediante el instrumento AC-OK estaban en tratamiento. Solo el 11,1\% (22 mujeres) habían recibido tratamiento en los servicios de salud mental o drogadicción en los últimos tres meses y el 13,6\% (27 mujeres) tenían cita para el mes siguiente. Este hallazgo resalta la importante falta de atención en salud mental y adicciones durante el embarazo y sugiere que se deben establecer nuevas acciones que permitan a los profesionales involucrados en la atención de la embarazada reconocer el uso de sustancias y los trastornos de salud mental, y brindar las derivaciones adecuadas para su tratamiento.

A todas las mujeres embarazadas se les debe preguntar regularmente sobre el consumo de sustancias (Siu, 2015). Las bajas tasas de tratamiento en nuestra muestra sugieren que no todos los obstetras, ni otros profesionales involucrados en el tratamiento de mujeres embarazadas, están identificando el uso de sustancias y los problemas de salud mental. Dadas las consecuencias del uso de sustancias, debería ser parte de la atención habitual, no solo en la primera visita, sino también a lo largo de las siguientes citas, para evaluar y promover el cese del consumo. Varias razones pueden estar detrás de esta falta de reconocimiento e identificación adecuados del trastorno por uso de sustancias y las enfermedades mentales. Entre ellas, y sumado a la frecuente infradeclaración, podría estar el desconocimiento del alcance de la repercusión en el feto, que probablemente esté subestimado a corto y largo plazo. Es más, por otro lado, como se ha descrito en la literatura (Ebrahim y Gfroerer, 2003), el perfil de la paciente suele ser más complejo, siendo habitualmente más joven, con un nivel socioeconómico bajo y mayores tasas de control gestacional inadecuado. Nuestros hallazgos de resultados positivos más altos en el AC-OK en aquellas pacientes con niveles educativos más bajos apoyan esta hipótesis. Además, estas pacientes también pueden negar su consumo para evitar las repercusiones legales que a menudo se asocian con la divulgación.

La anamnesis es la herramienta esencial para identificar a pacientes con problemas de salud mental y uso de sustancias, pero son limitadas las herramientas de cribado validadas para su uso en mujeres embarazadas (ACOG Committee Opinion No. 524: Opioid abuse, dependence, and addiction in pregnancy, 2012). Una de estas herramientas es el CRAFFT, que se ha informado que es mejor que el informe médico y la prueba de detección de alcohol T-ACE para la identificación de pacientes jóvenes (menores de 25 años) que consumen sustancias prenatales, pero no se ha validado en pacientes mayores (Chang et al., 2011). También ha de considerarse si, debido a la habitual falta de tiempo de las citas médicas, se deberían aplicar otras herramientas de cribado adicionales de forma universal o solo en aquellas pacientes que responden afirmativamente a preguntas sobre consumo. Además, el uso de pruebas biológicas como herramienta de cribado podría mejorar la detección y minimizar la infradeclaración, pero sería menos factible usarlas en la atención de rutina (Garg et al., 2016). Un cuestionario muy simple como el AC-OK parece prometedor y podría ser adecuado para una identificación precisa en la atención habitual de las mujeres embarazadas.

Hay varias limitaciones a tener en cuenta al sacar conclusiones de los resultados obtenidos. Primero, a la hora de evaluar los resultados de prevalencia en el embarazo, hay que considerar que la muestra no se extrae de toda la región de Madrid donde están ubicados los hospitales. Segundo, una posible subestimación de la prevalencia, dada la infradeclaración habitual del consumo de sustancias y de problemas de salud mental, puede afectar las cifras (Garg et al., 2016).

A pesar de estas limitaciones, se puede concluir que, debido a la combinación de prevalencias significativas, las bajas tasas de tratamiento y las graves, pero prevenibles consecuencias sobre la madre y el niño, nuevas acciones, incluyendo mecanismos de detección eficientes, deben integrarse en la práctica clínica habitual. Esto permitiría un acceso adecuado al tratamiento y la promoción del cese temprano del consumo de sustancias.

\section{Reconocimientos}

Este estudio se ha realizado en el marco del proyecto PSI2016- 75854-P del Ministerio de Economía y Empresa de España.

Este estudio contó con el apoyo de la Delegación del Gobierno para el Plan Nacional sobre Drogas, de la Secretaría de Estado de Servicios Sociales e Igualdad del Ministerio de Sanidad, Consumo y Bienestar Social (20151073).

\section{Conflicto de intereses}

Los autores declaran la inexistencia de conflicto de interés respecto de cualquier aspecto de este estudio.

Los autores declaran la inexistencia de conflicto de interés en relación al estudio, su autoría y/o la publicación de este manuscrito.

\section{Referencias}

ACOG Committee Opinion No. 524: Opioid abuse, dependence, and addiction in pregnancy. (2012). Obstet Gynecol, 119, 1070-1076. doi:10.1097/AOG.0b013e318256496e. 
Austin, M. P., Priest, S. R. y Sullivan, E. A. (2008). Antenatal psychosocial assessment for reducing perinatal mental health morbidity. Cochrane Database Systematic Revision, 4, CD005124. doi:10.1002/14651858.CD005124.pub2.

Barrigon, M. L., Berrouiguet, S., Carballo, J. J., Bonal-Gimenez, C., Fernandez-Navarro, P., Pfang, B.,... Baca-Garcia, E. (2017). User profiles of an electronic mental health tool for ecological momentary assessment: MEmind. International Journal of Methods in Psychiatric Research, 26, e1554. doi:10.1002/mpr.1554.

Bayrampour, H., Hapsari, A. P. y Pavlovic, J. (2018). Barriers to addressing perinatal mental health issues in midwifery settings. Midwifery, 59, 47-58. doi:10.1016/j. midw.2017.12.020.

Blanchard, E. B., Jones-Alexander, J., Buckley, T. C. y Forneris, C. A. (1996). Psychometric properties of the PTSD Checklist (PCL). Behaviour Research and Therapy, 34, 669-673.

Blasco-Alonso, M., Gonzalez-Mesa, E., Galvez Montes, M., Lozano Bravo, I., Merino Galdon, F., Cuenca Campos, F.,... Bellido Estevez, I. (2015). Exposure to tobacco, alcohol and drugs of abuse during pregnancy. A study of prevalence among pregnant women in Malaga (Spain). Adicciones, 27, 99-108.

Blatt, K., Moore, E., Chen, A., Van Hook, J. y DeFranco, E. A. (2015). Association of reported trimester-specific smoking cessation with fetal growth restriction. Obstetrics E于 Gynecology, 125, 1452-1459. doi:10.1097/ aog.0000000000000679.

Bohn, M. J., Babor, T. F. y Kranzler, H. R. (1995). The Alcohol Use Disorders Identification Test (AUDIT): Validation of a screening instrument for use in medical settings. Journal of Studies on Alcohol, 56, 423-432. doi:10.15288/jsa.1995.56.423.

Cnattingius, S. (2004). The epidemiology of smoking during pregnancy: Smoking prevalence, maternal characteristics, and pregnancy outcomes. Nicotine E Tobacco Reserach, 6 (Supl. 2), S125-140. doi:10.1080/146222004 10001669187.

Cook, J. L., Green, C. R., de la Ronde, S., Dell, C. A., Graves, L., Ordean, A.,... Wong, S. (2017). Epidemiology and effects of substance use in pregnancy. Journal of Obstetrics and Gynaecology Canada, 39, 906-915. doi:10.1016/j. jogc.2017.07.005.

Chang, G., Orav, E. J., Jones, J. A., Buynitsky, T., Gonzalez, S. y Wilkins-Haug, L. (2011). Self-reported alcohol and drug use in pregnant young women: A pilot study of associated factors and identification. Journal of Addiction Medicine, 5, 221-226. doi:10.1097/ADM. 0b013e318214360b.

Chavez, L. M., Shrout, P. E., Wang, Y., Collazos, F., Carmona, R. y Alegria, M. (2017). Evaluation of the AC-OK mental health and substance abuse screening measure in an international sample of Latino immigrants. Drug and Alcohol Dependence, 180, 121-128. doi:10.1016/j.drugalcdep.2017.07.042.

Cherry, A. L. y Dillon, M. E. (2013). The AC-OK cooccurring screen: Reliability, convergent validity, sensitivity, and specificity. Journal of Addiction, 2013, 573906. doi:10.1155/2013/573906.

Dahlin, S., Gunnerbeck, A., Wikstrom, A. K., Cnattingius, S. y Edstedt Bonamy, A. K. (2016). Maternal tobacco use and extremely premature birth - a population-based cohort study. British Journal of Obstetrics and Gynaecology, 123, 1938-1946. doi:10.1111/1471-0528.14213.

Donald, K. A., Eastman, E., Howells, F. M., Adnams, C., Riley, E. P., Woods, R. P.,... Stein, D. J. (2015). Neuroimaging effects of prenatal alcohol exposure on the developing human brain: A magnetic resonance imaging review. Acta Neuropsychiatrica, 27, 251-269. doi:10.1017/ neu.2015.12.

Ebrahim, S. H. y Gfroerer, J. (2003). Pregnancy-related substance use in the United States during 1996-1998. Obstetrics Ev Gynecology, 101, 374-379.

Ekblad, M., Gissler, M., Lehtonen, L. y Korkeila, J. (2010). Prenatal smoking exposure and the risk of psychiatric morbidity into young adulthood. Archives of General Psychiatry, 67, 841-849. doi:10.1001/archgenpsychiatry.2010.92.

England, M. C., Benjamin, A. y Abenhaim, H. A. (2013). Increased risk of preterm premature rupture of membranes at early gestational ages among maternal cigarette smokers. American Journal of Perinatology, 30, 821-826. doi:10.1055/s-0032-1333408.

Fairbrother, N., Janssen, P., Antony, M. M., Tucker, E. y Young, A. H. (2016). Perinatal anxiety disorder prevalence and incidence. Journal of Affective Disorders, 200, 148-155. doi:10.1016/j.jad.2015.12.082.

Forray, A. y Foster, D. (2015). Substance use in the perinatal period. Current Psychiatry Reports, 17, 91. doi:10.1007/ s11920-015-0626-5.

Garg, M., Garrison, L., Leeman, L., Hamidovic, A., Borrego, M., Rayburn, W. F. y Bakhireva, L. (2016). Validity of self-reported drug use information among pregnant women. Maternal and Child Health Journal, 20, 41-47. doi:10.1007/s10995-015-1799-6.

Gauthier, T. W., Guidot, D. M., Kelleman, M. S., McCracken, C. E. y Brown, L. A. (2016). Maternal alcohol use during pregnancy and associated morbidities in very low birth weight newborns. American Journal of the Medical Sciences, 352, 368-375. doi:10.1016/j.amjms.2016.06.019.

Gobierno de España. Ley Orgánica 3/2018 de Protección de Datos Personales y garantía de los derechos digitales. (2018). Madrid, Spain: Recuperado de https://www. boe.es/eli/es/lo/2018/12/05/3/dof/spa/pdf.

Gouin, K., Murphy, K. y Shah, P. S. (2011). Effects of cocaine use during pregnancy on low birthweight and preterm birth: Systematic review and metaanalyses. Ameri- 
can Journal of Obstetrics $\mathcal{E}$ Gynecology, 204, 340 e341-312. doi:10.1016/j.ajog.2010.11.013.

Hankin, J., McCaul, M. E. y Heussner, J. (2000). Pregnant, alcohol-abusing women. Alcoholism: Clinical and Experimental Research, 24, 1276-1286.

Heatherton, T. F., Kozlowski, L. T., Frecker, R. C. y Fagerstrom, K. O. (1991). The Fagerstrom test for nicotine dependence: A revision of the Fagerstrom tolerance questionnaire. British Journal of Addiction, 86, 1119-1127.

Holbrook, B. D. y Rayburn, W. F. (2014). Teratogenic risks from exposure to illicit drugs. Obstetrics and Gynecology Clinics of North America, 41, 229-239. doi:10.1016/j. ogc.2014.02.008.

Howard, L. M., Piot, P. y Stein, A. (2014). No health without perinatal mental health. Lancet, 384, 1723-1724. doi:10.1016/s0140-6736(14)62040-7.

Kingston, D., Tough, S. y Whitfield, H. (2012). Prenatal and postpartum maternal psychological distress and infant development: A systematic review. Child Psychiatry Ẽ Human Development, 43, 683-714. doi:10.1007/s10578012-0291-4

Ko, T. J., Tsai, L. Y., Chu, L. C., Yeh, S. J., Leung, C., Chen, C. Y.,... Hsieh, W. S. (2014). Parental smoking during pregnancy and its association with low birth weight, small for gestational age, and preterm birth offspring: A birth cohort study. Pediatrics and Neonatology, 55, 20-27. doi:10.1016/j.pedneo.2013.05.005.

Kramer, M. S., Lydon, J., Seguin, L., Goulet, L., Kahn, S. R., McNamara, H.,... Platt, R. W. (2009). Stress pathways to spontaneous preterm birth: The role of stressors, psychological distress, and stress hormones. American Journal of Epidemiology, 169, 1319-1326. doi:10.1093/aje/kwp061.

Kroenke, K., Spitzer, R. L. y Williams, J. B. (2001). The PHQ-9: Validity of a brief depression severity measure. Journal of General Internal Medicine, 16, 606-613.

Lange, S., Probst, C., Rehm, J. y Popova, S. (2018). National, regional, and global prevalence of smoking during pregnancy in the general population: A systematic review and meta-analysis. Lancet Global Health, 6, e769-e776. doi:10.1016/s2214-109x(18)30223-7.

Martinez-Paredes, J. F. y Jacome-Perez, N. (2019). Depression in pregnancy. Revista Colombiana de Psiquiatría, 48, 58-65. doi:10.1016/j.rcp.2017.07.003.
Paykel, E. S., Myers, J. K., Lindenthal, J.J. y Tanner, J. (1974). Suicidal feelings in the general population: A prevalence study. British Journal of Psychiatry, 124, 460-469.

Pereira, P. P., Da Mata, F. A., Figueiredo, A. C., de Andrade, K. R. y Pereira, M. G. (2017). Maternal active smoking during pregnancy and low birth weight in the americas: A systematic review and meta-analysis. Nicotine Eo Tobacco Research, 19, 497-505. doi:10.1093/ntr/ntw228.

Pineles, B. L., Hsu, S., Park, E. y Samet, J. M. (2016). Systematic review and meta-analyses of perinatal death and maternal exposure to tobacco smoke during pregnancy. American Journal of Epidemiology, 184, 87-97. doi:10.1093/ aje/kwv301.

Sarman, I. (2018). Review shows that early foetal alcohol exposure may cause adverse effects even when the mother consumes low levels. Acta Paediatrica, 107, 938-941. doi:10.1111/apa.14221.

Siu, A. L. (2015). Behavioral and pharmacotherapy interventions for tobacco smoking cessation in adults, including pregnant women: U.S. preventive services Task Force Recommendation Statement. Annals of Internal Medicine, 163, 622-634. doi:10.7326/m15-2023.

Spitzer, R. L., Kroenke, K., Williams, J. B. y Lowe, B. (2006). A brief measure for assessing generalized anxiety disorder: The GAD-7. Archives of Internal Medicine, 166, 10921097. doi:10.1001/archinte.166.10.1092.

Tiesler, C. M. y Heinrich, J. (2014). Prenatal nicotine exposure and child behavioural problems. European Child $\mathcal{E}$ Adolescent Psychiatry, 23, 913-929. doi:10.1007/s00787014-0615-y.

Woody, C. A., Ferrari, A. J., Siskind, D. J., Whiteford, H. A. y Harris, M. G. (2017). A systematic review and meta-regression of the prevalence and incidence of perinatal depression. Journal of Affective Disorders, 219, 86-92. doi:10.1016/j.jad.2017.05.003.

Yudko, E., Lozhkina, O. y Fouts, A. (2007). A comprehensive review of the psychometric properties of the Drug Abuse Screening Test. Journal of Substance Abuse Treatment, 32, 189-198. doi:10.1016/j.jsat.2006.08.002. 
